Pacific Journal of Mathematic 


\section{SOME APPLICATIONS OF ULTRAFILTERS IN TOPOLOGY}

\section{John Ginsburg AND VICTOR SAKS}

In this paper, those Hausdorff spaces, all of whose powers are countably compact, are characterized, and partial results on the corresponding question for pseudocompactness are obtained. The basic tool in this work is A. R. Bernstein's concept of $\mathscr{D}$-compactness. Sufficient conditions are found for every power of a topological group to be countably compact. The maximal $\mathscr{D}$-compact extension of a completely regular space is constructed, and this procedure is used to construct the unique $\mathscr{D}$-compact group extension of a totally bounded topological group. Additional product theorems for pseudocompact spaces are proved, imposing conditions closely related to $\mathscr{D}$. compactness on the factors, which imply the pseudocompactness of the product. In the final section of the paper, several theorems are proved which provide new examples of nontrivial pseudocompact spaces. In particular, a homogeneous space is exhibited, all of whose powers are pseudocompact, in which no discrete countable set has a cluster point.

All spaces in this paper are assumed to be Hausdorff, and this assumption will be used without explicit mention. We shall use throughout the terminology and notation of the Gillman and Jerison text [10]. In particular, the set of positive integers is denoted by $N$, and the cardinality of a set $S$ is denoted by $|S|$. The Stone-Čech compactification of a completely regular space $X$ is denoted by $\beta X$. The symbol $[\mathrm{CH}]$ indicates that the continuum hypothesis is being assumed.

2. Countably compact powers. Let us recall several definitions of compactness-like conditions which depend on the behaviour of countable sets.

Let $X$ be a topological space.

$X$ is said to be countably compact, if every countably infinite subset of $X$ has a cluster point.

A subset $A$ of $X$ is relatively countably compact in $X$, if every countably infinite subset of $A$ has a cluster point in $X$.

$X$ is sequentially compact, if every sequence in $X$ has a convergent subsequence. 
$X$ is called strongly $\boldsymbol{\aleph}_{0}$-compact, if every infinite subset of $X$ meets some compact subset of $X$ in an infinite set.

Finally, we call $X \boldsymbol{\aleph}_{0}$-bounded, if every countable subset of $X$ is contained in a compact subset of $X$.

Our first result characterizes those spaces $X$ such that every power of $X$ is countably compact. The main tool in this investigation is Bernstein's concept of $\mathscr{D}$-compactness. In [1] the concept was introduced, and some of the basic theory of $\mathscr{D}$-compact spaces was developed. We now give his definition of $\mathscr{D}$-compactness, and quote the major results in [1], including a proof of his result that $\mathscr{D}$ compactness is a productive property.

Definition 2.1. Let $\mathscr{D}$ be a free ultrafilter on $N$. Let $X$ be a topological space, and let $\left(x_{n}: n \in N\right)$ be a sequence in $X$. A point $z \in X$ is said to be a $\mathscr{D}$-limit point of the sequence $\left(x_{n}: n \in N\right)$ if, for every neighborhood $W$ of $z,\left\{n: x_{n} \in W\right\} \in \mathscr{D}$. $\mathscr{D}$-limits, when they exist, are unique, and we shall express the fact that $z$ is the $\mathscr{D}$-limit of $\left(x_{n}: n \in N\right)$ by writing $z=\mathscr{D}-\lim _{n \rightarrow \infty} x_{n}$. A space $X$ is said to be $\mathscr{D}$-compact if every sequence in $X$ has a $\mathscr{D}$-limit point.

Observe that a $\mathscr{D}$-limit point of a sequence of distinct points $\left(x_{n}: n \in N\right)$ is, in particular, a cluster point of the set $\left\{x_{n}: n \in N\right\}$. Therefore a $\mathscr{D}$-compact space is countably compact.

LEMmA 2.2. Let $\left\{x_{n}: n \in N\right\} \subseteq X$ and let $z \in X$ be a cluster point of $\left\{x_{n}: n \in N\right\}$. Then there exists $\mathscr{D}$ in $\beta N-N$ such that $z=$ $\mathscr{D}-\lim _{n \rightarrow \infty} x_{n}$.

Proof. Let $\mathscr{G}(z)$ denote the family of all neighborhoods of $z$ in $X$. For $W \in \mathscr{G}(z)$, let $s(W)=\left\{n: x_{n} \in W\right\}$. The family $\mathscr{F}=\{s(W\}-$ $\{k\}: W \in \mathscr{G}(z), k \in N\}$ has the finite intersection property, and so there is an ultrafilter $\mathscr{D}$ on $N$ such that $\mathscr{F} \subseteq \mathscr{D}$. Obviously $\mathscr{D}$ is free and $z=\mathscr{D}-\lim _{n \rightarrow \infty} x_{n}$.

Lemma 2.3. Let $f: X \rightarrow Y$ be a continuous map. Let $\left(x_{n}: n \in N\right)$ be a sequence in $X$, and let $z \in X$ such that $z=\mathscr{D}-\lim _{n \rightarrow \infty} x_{n}$. Then $f(z)=\mathscr{D}-\lim _{n \rightarrow \infty} f\left(x_{n}\right)$.

Proof. For every neighborhood $W$ of $f(z)$ in $Y, f^{-1}(W)$ is a neighborhood of $z$ in $X$. Since $\left\{n: x_{n} \in f^{-1}(W)\right\}=\left\{n: f\left(x_{n}\right) \in W\right\}$, the result follows. 
TheOREM 2.4 (Bernstein). D-compactness is closed hereditary and productive. A completely regular space is $\boldsymbol{\aleph}_{0}$-bounded if, and only if, it is $\mathscr{D}$-compact for every $\mathscr{D}$ in $\beta N-N$.

Proof. Obviously a closed subset of a $\mathscr{D}$-compact space is $\mathscr{D}$ compact. We will prove the statement concerning products, and refer the reader to Theorems 3.4 and 3.5 of [1] for the last statement.

Thus, let $\left\{X_{\alpha}: \alpha \in I\right\}$ be a family of $\mathscr{D}$-compact spaces, and let $X=\prod_{\alpha \in I} X_{\alpha}$. We will show that $X$ is $\mathscr{D}$-compact. Let $\left(x^{(n)}: n \in N\right)$ be a sequence in $X$. Then, for each $\alpha$ in $I,\left(x_{\alpha}^{(n)}: n \in N\right)$ has a $\mathscr{D}$ limit point $z_{\alpha}$ in $X_{\alpha}$. This defines a point $z=\left(z_{\alpha}\right)_{\alpha \in I}$ in $X$. We claim that $z=\mathscr{D}-\lim _{n \rightarrow \infty} x^{(n)}$. For, let $W$ be any neighborhood of $z$ in $X$. There is a finite subset $F$ of $I$, and open sets $W_{\alpha}$ in $X_{\alpha}$, for each $\alpha$ in $F$, such that $z \in \Pi_{\alpha \in F} W_{\alpha} \times \prod_{\alpha \notin F} X_{\alpha} \subseteq W$.

But $\left\{n: x^{(n)} \in W\right\} \supseteqq \bigcap_{\alpha \in F}\left\{n: x_{\alpha}{ }^{(n)} \in W_{\alpha}\right\}$, and therefore $\left\{n: x^{(n)} \in\right.$ $W\} \in \mathscr{D}$. This proves that $z=\mathscr{D}-\lim _{n \rightarrow \infty} x^{(n)}$ and thus $X$ is $\mathscr{D}$-compact.

CoROLlaRY 2.5. Any product of $\mathscr{D}$-compact spaces is countably compact.

Proof. Immediate.

We are now in a position to characterize spaces all of whose powers are countably compact.

THEOREM 2.6. Let $X$ be a Hausdorff space. The following statements are equivalent:

(i) Every power of $X$ is countably compact;

(ii) $X^{2^{c}}$ is countably compact;

(iii) $X^{|X| \aleph_{0}}$ is countably compact;

(iv) There exists $\mathscr{D}$ in $\beta N-N$ such that $X$ is $\mathscr{D}$-compact.

Proof. (i ) $\Rightarrow$ (ii). This is trivial.

(ii) $\Rightarrow$ (iv). We show that if (iv) fails, so does (ii). Thus, suppose $X$ is not $\mathscr{D}$-compact for any $\mathscr{D}$ in $\beta N-N$. Then, for each $\mathscr{D}$ in $\beta N-N$, there is a sequence $\left(x_{n}^{\mathscr{D}}: n \in N\right)$ in $X$ which has no $\mathscr{D}$-limit point in $X$. Define a sequence $\left(y^{(n)}: n \in N\right)$ in $X^{\beta N-N}$ as follows: $y_{\mathscr{D}}^{(n)}=n_{n}$.

For the sake of contradiction, assume (ii) holds. Then $X^{\beta N-N}$ is countably compact, and therefore the sequence $\left(y^{(n)}: n \in N\right)$ has a cluster point $z$ in $X^{\beta N-N}$. By Lemma 2.2, there exists $\mathscr{E}$ in $\beta N-N$ such that $z=\mathscr{E}-\lim _{n \rightarrow \infty} y^{(n)}$. But this implies, by Lemma 2.3, that 


$$
\Pi_{\mathscr{E}}(z)=\mathscr{E}-\lim _{n \rightarrow \infty} \Pi_{\mathscr{E}}\left(y^{(n)}\right)=\mathscr{E}-\lim _{n \rightarrow \infty} x_{n}^{(\mathscr{8})} .
$$

But this is ridiculous, since $\left(x_{n}^{(8)}: n \in N\right)$ has no $\mathscr{E}$-limit point. Thus (ii) must also fail.

(iv) $\Rightarrow$ (i ). This follows immediately from 2.5.

(i) $\Rightarrow$ (iii). This is trivial.

(iii) $\Rightarrow$ (iv). Let $\Sigma$ be the set of all sequences in $X$. We write $\sigma \in \Sigma$ as $\sigma=\left(x_{n}^{(\sigma)}: n \in N\right)$. Now $|\Sigma|=|X|^{\boldsymbol{\aleph}_{0}}$, and so (iii) implies that $X^{\Sigma}$ is countably compact. Define a sequence $\left(z^{(n)}: n \in N\right)$ in $X^{\Sigma}$ as follows: $z_{\sigma}^{(m)}=x_{m}^{(o)}$. Let $p \in X^{\Sigma}$ be a cluster point of $\left(z^{(n)}: n \in N\right)$. By 2.2, there exists $\mathscr{D}$ in $\beta N-N$ such that $p=\mathscr{D}-\lim _{n \rightarrow \infty} z^{(n)}$.

We claim that, for this $\mathscr{D}, X$ is $\mathscr{D}$-compact. For, if $\sigma=$ $\left(x_{n}^{(o)}: n \in N\right)$ is any sequence in $X$, Lemma 2.3 implies that $\Pi_{o}(p)=$ $\mathscr{D}-\lim _{n \rightarrow \infty} \Pi_{o}\left(z^{(n)}\right)=\mathscr{D}-\lim _{n \rightarrow \infty} x_{n}^{(\sigma)}$. Thus every sequence in $X$ has a $\mathscr{D}$-limit point, and so $X$ is $\mathscr{D}$-compact.

REMARK 2.7. In [19], Scarborough and Stone have shown that, if $X=\prod_{\alpha \in I} X_{\alpha}$, then $X$ is countably compact if, and only if, every subproduct of $2^{2^{c}}$ factors is countably compact. Thus the conditions (ii) and (iii) in Theorem 2.6 may be regarded as an improvement of their result in the case where all the factors are the same.

CoROLlary 2.8. If $|X| \leqq c$, then $X^{c}$ is countably compact if, and only if, there exists $\mathscr{D}$ in $\beta N-N$ such that $X$ is $\mathscr{D}$-compact.

In [18] the following theorem is proved.

THEOREM 2.9 (Saks-Stephenson). The product of not more than $\aleph_{1}$ strongly $\aleph_{0}$-compact spaces is countably compact.

We therefore obtain the following corollary.

CoROLlary 2.10. [CH] If $|X| \leqq c$, and if $X$ is strongly $\aleph_{0^{-}}$ compact, then there exists $\mathscr{D}$ in $\beta N-N$ such that $X$ is $\mathscr{D}$-compact. In particular, every countably compact $k$-space of cardinality $\leqq c$ is $\mathscr{D}$-compact for some $\mathscr{D}$ in $\beta N-N$.

Proof. The first assertion is obvious from 2.8 and 2.9, while the second is a special case, by Theorem 1.2 in [15].

REMARK 2.11. Since every sequentially compact space is strongly $\aleph_{0}$-compact, the conclusion of 2.10 holds for sequentially compact spaces, of cardinal $\leqq c$. This special case of 2.10 also follows directly 
from Theorem 5.8 in [19], together with our Theorem 2.6.

For non-trivial examples of the spaces hypothesized in 2.10 , the reader is referred to [6].

We now turn to another aspect of $\mathscr{D}$-compactness. It follows from the corollary to Theorem 1 in [12], that every completely regular space has a maximal $\mathscr{D}$-compact extension. That is, for every completely regular space $X$, there is a $\mathscr{D}$-compact space $\mathscr{D}(X)$ containing $X$ as a dense subspace, such that every continuous map of $X$ into any $\mathscr{D}$-compact space extends continuously to $\mathscr{D}(X)$. From the final section of Herrlich and van der Slot's paper [12], it follows that, in fact, we may take $X \subseteq \mathscr{D}(X) \subseteq \beta X$ where $\mathscr{D}(X)$ is the intersection of all $\mathscr{D}$-compact subspaces of $\beta X$ containing $X$.

We now show how $\mathscr{D}(X)$ is built up from $X$. The construction is an exact analogue of Example 4 in [1]. In this example, Bernstein is constructing a $\mathscr{D}$-compact space which is not $\boldsymbol{\aleph}_{0}$-bounded. His construction, when slightly modified, gives the maximal $\mathscr{D}$-compact extension of an arbitrary completely regular space. R. G. Woods independently characterized $\mathscr{D}(X)$ by the same method as given here, in [22].

Let $X$ be a completely regular space. We first construct a transfinite sequence $\left(X_{\alpha}: \alpha<\omega_{1}\right)$ of subspaces of $\beta X$ containing $X$.

Let $X_{0}=X$. Assume we have constructed the spaces $X_{\alpha}$, for $\alpha<\beta$ such that

(i ) $\alpha_{1} \leqq \alpha_{2}<\beta \Rightarrow X_{\alpha_{1}} \subseteq X_{\alpha_{2}} \subseteq \beta X$

(ii) $\alpha_{1}<\alpha_{2}<\beta \Rightarrow$ every sequence in $X_{\alpha_{1}}$ has a $\mathscr{D}$-limit point in $X_{\alpha_{2}}$.

We now show how to get $X_{\beta}$. Let $\Sigma_{\beta}$ be the set of all sequences in $\mathrm{U}_{\alpha<\beta} X_{\alpha}$. For each $\sigma \in \Sigma_{\beta}$, let $x_{\sigma}$ be the $\mathscr{D}$-limit point of $\sigma$ in $\beta X$. Finally, let $X_{\beta}=\left(\bigcup_{\alpha<\beta} X_{\alpha}\right) \cup\left\{x_{\sigma}: \sigma \in \Sigma_{\beta}\right\}$.

This completes the induction step, and gives a sequence $\left(X_{\alpha}: \alpha<\omega_{1}\right)$ satisfying (i) and (ii) for all $\alpha_{1}<\alpha_{2}<\omega_{1}$.

\section{THEOREM 2.12. $\mathscr{D}(X)=\bigcup_{\alpha<\omega_{1}} X_{\alpha}$.}

Proof. Obviously $X \subseteq \mathrm{U}_{\alpha<\omega_{1}} X_{\alpha} \subseteq \beta X$. If $\left(x_{n}: n \in N\right)$ is a sequence in $\mathrm{U}_{\alpha<\omega_{1}} X_{\alpha}$, it lies entirely within one $X_{\beta}$, and thus has a $\mathscr{D}$-limit point in $X_{\beta+1}$. Therefore $\mathrm{U}_{\alpha<\omega} X_{\alpha}$ is $\mathscr{D}$-compact. A straightforward induction shows that any $\mathscr{D}$-compact subspace of $\beta X$ containing $X$ 
must contain every $X_{\alpha}$, that is, must contain $\mathrm{U}_{\alpha<\omega_{1}} X_{\alpha}$. From the result we have quoted from the Herrlich and van der Slot paper [12], it now follows that $\mathscr{D}(X)=\bigcup_{\alpha<\omega_{1}} X_{\alpha}$.

3. $\mathscr{D}$-compactness in topological groups. Recall that a topological group $G$ is said to be totally bounded, if, for each neighborhood $U$ of the identity, a finite number of translates of $U$ covers $G$. In [20], Weil proves that each totally bounded group $G$ is a dense topological subgroup of a compact group, and that this compactification is unique up to a topological isomorphism fixing $G$ pointwise. This compactification of $G$ is known as its Weil completion, and shall be denoted by $\bar{G}$.

Using the identical procedure to 2.12 , we obtain the following result. Let $\mathscr{D}$ be a free ultrafilter on $N$.

THEOREM 3.1. For every totally bounded topological group $G$, there is a $\mathscr{D}$-compact topological group $\mathscr{D}(G)$, containing $G$ as a dense topological subgroup, such that no proper subspace of $\mathscr{D}(G)$ containing $G$ is $\mathscr{D}$-compact. $\mathscr{D}(G)$ is determined uniquely by a topological isomorphism fixing $G$ pointwise.

Proof. Define the spaces $\left(G_{\alpha}: \alpha<\omega_{1}\right)$ as in 2.12 , replacing $X$ by $G$, and $\beta X$ by $\bar{G}$, and let $\mathscr{D}(G)=\bigcup_{\alpha<\omega_{1}} G_{\alpha} . \mathscr{D}(G)$ is $\mathscr{D}$-compact and there is, as in 2.12, no $\mathscr{D}$-compact space $Y$ satisfying, $G \cong Y \subset$ $\mathscr{D}(G)$. We next show that $\mathscr{D}(G)$ is a group. Since the $G_{\alpha}$ form a chain, to prove this, it is sufficient to prove the following:

If $H$ is a subgroup of $\bar{G}$, and if $L_{H}=\{x \in \bar{G}$ : there exists a sequence $\left(x_{n}: n \in N\right)$ in $H$ such that $\left.x=\mathscr{D}-\lim _{n \rightarrow \infty} x_{n}\right\}$, then $H \cup L_{H}$ is a subgroup of $\bar{G}$. But this is obvious, since if $x=\mathscr{D}-\lim _{n \rightarrow \infty} x_{n}$ and $y=\mathscr{D}-\lim _{n \rightarrow \infty} y_{n}$, then the continuity of the operations implies that $x^{-1}=\mathscr{D}-\lim _{n \rightarrow \infty} x_{n}^{-1}$ and $x y=\mathscr{D}-\lim _{n \rightarrow \infty} x_{n} y_{n}$.

We have now shown that $\mathscr{D}(G)$ is a $\mathscr{D}$-compact group containing $G$ as a dense topological subgroup, and that $\mathscr{D}(G)$ is a minimal $\mathscr{D}$ compact extension of $G$. It remains to prove the uniqueness assertion.

Recall that, in Theorem 1.1 of [3], Comfort and Ross prove that every pseudocompact group is totally bounded. In particular, any $\mathscr{D}$-compact group is totally bounded.

Now, suppose $H$ is another $\mathscr{D}$-compact group containing $G$ as a dense topological subgroup, and that no proper subspace of $H$ 
containing $G$ is $\mathscr{D}$-compact. Since $H$ is totally bounded, it has a Weil completion, $\bar{H}$, and by uniqueness, $\bar{G} \simeq \bar{H}$, by a topological isomorphism fixing $G$ pointwise. By the construction of $\mathscr{D}(G)$ it is clear that under any such isomorphism, $\mathscr{D}(G)$ is mapped inside $H$. Since this image of $\mathscr{D}(G)$ is $\mathscr{D}$-compact and contains $G$, the minimality of $H$ implies that the image of $\mathscr{D}(G)$ under any such isomorphism is all of $H$. This proves that $\mathscr{D}(G)$ is uniquely determined, by a topological isomorphism fixing $G$ pointwise, as a minimal $\mathscr{D}$-compact group extension of $G$.

Our next result gives conditions sufficient for a topological group to be $\mathscr{D}$-compact for some $\mathscr{D}$ in $\beta N-N$.

THEOREM 3.2. Let $G$ be a countably compact group. If $G$ contains a $C^{*}$-embedded copy of $N$, and if multiplication $m: G \times G \rightarrow G$ is a closed map, then $G$ is $\mathscr{D}$-compact for some $\mathscr{D}$ in $\beta N-N$.

Proof. We will identify the $C^{*}$-embedded copy of $N$ as being $N$ itself. In Theorem 1.2 of [3], Comfort and Ross prove that, if $G$ is a pseudocompact group, then $\bar{G}=\beta G$. Therefore, under the given hypotheses on $G, \operatorname{cl}_{\bar{G}} N=\beta N$.

Since $G$ is countably compact, $N$ has a cluster point $\mathscr{D} \in G \cap \beta N=$ $G \cap \mathrm{cl}_{\bar{G}} N$. We will show that $G$ is $\mathscr{D}$-compact.

Thus, let $\left(b_{n}: n \in N\right)$ be a sequence in $G$. We must find a $\mathscr{D}$-limit point of $\left(b_{n}: n \in N\right)$ lying in $G$. Consider the sequence $\left(\left(b_{n}, b_{n}^{-1} n\right): n \in N\right)$ in $G \times G$. Since $\mathscr{D} \in \operatorname{cl}_{G} m\left(\left\{\left(b_{n}, b_{n}^{-1} n\right): n \in N\right\}\right)$, and since multiplication is closed, there exists $b \in G$ such that $\left(b, b^{-1} \mathscr{D}\right) \in \operatorname{cl}_{G \times G}\left\{\left(b_{n}, b_{n}^{-1} n\right): n \in N\right\}$. We show that, in fact, $b=\mathscr{D}-\lim _{n \rightarrow \infty} b_{n}$. For the sake of contradiction, assume $b$ is not the $\mathscr{D}$-limit point of $\left(b_{n}: n \in N\right)$. Then there is a neighborhood $U$ of the identity in $G$ such that $L=\left\{n: b_{n} \in b U\right\} \notin \mathscr{D}$. Let $K=N-L$. Then $K \in \mathscr{D}$. Thus $\mathscr{D} \in \mathrm{cl}_{\beta N} K=\operatorname{cl}_{\bar{G}} K$, and $\operatorname{so~}_{\bar{G}} K$ is a neighborhood of $\mathscr{D}$ in $\beta N=\operatorname{cl}_{\bar{G}} N$. Therefore there is an open set $\bigcirc$ in $\bar{G}$ such that $\bigcirc \cap \beta N=\operatorname{cl}_{\bar{G}} K$. Let $V=\mathscr{D}^{-1}(\bigcirc \cap G)$. Then $V$ is a neighbborhood of the identity in $G$, and $\mathscr{D} V \cap N=K$. Now find a neighorhood $W$ of the identity in $G$, such that $W^{2} \cong U \cap V$, and find a neighborhood $M$ of the identity in $G$ such that $M \subseteq U$ and $M b^{-1} \mathscr{D} \subseteq b^{-1} \mathscr{D} W$. Since $\left(b, b^{-1} \mathscr{D}\right) \in \mathrm{cl}_{G \times G}\left\{\left(b_{n}, b_{n}^{-1} n\right): n \in N\right\}$, there exists $n_{0}$ such that $\left(b_{n_{0}}, b_{n_{0}}^{-1} n_{0}\right) \in(b M) \times\left(b^{-1} \mathscr{D} W\right)$. Thus, $b_{n_{0}} \in b M \cong b U$, so that $n_{0} \in L$. But $n_{0}=\left(b_{n_{0}}\right)\left(b_{n_{0}}^{-1} n_{0}\right) \in(b M)\left(b^{-1} \mathscr{D} W\right)=b\left(M b^{-1} \mathscr{D}\right) W \subseteq$ $b\left(b^{-1} \mathscr{D} W\right) W=\mathscr{D} W^{2} \subseteq \mathscr{D} V$, and so $n_{0} \in \mathscr{D} V \cap N=K$. But this is impossible, since $K \cap L=\varnothing$. This contradiction proves that $b=$ $\mathscr{D}-\lim _{n \rightarrow \infty} b_{n}$, and thus $G$ is $\mathscr{D}$-compact. 
Observe that the proof of 3.2 actually shows that $G$ is $\mathscr{D}$-compact for all $\mathscr{D}$ in $G \cap \beta N$. This leads to the following corollary.

COROLlaRY 3.3. If $G$ is a topological group which contains a copy of $\beta N$, and if multiplication in $G$ is closed, then $G$ is $\boldsymbol{\aleph}_{0}^{-}$ bounded.

Proof. The proof of 3.2 shows that $G$ is $\mathscr{D}$-compact for all $\mathscr{D}$ in $\beta N-N$, and by Theorem 2.4, this is equivalent to $\aleph_{0}$-boundedness.

REMARK 3.4. Unfortunately, the authors know of no nontrivial groups on which multiplication is closed, aside from compact groups. Thus, as of now, Theorem 3.2 is not of practical use. In fact, it is still not known if there exist countably compact groups whose product is not countably compact. The corresponding question for pseudocompact groups has been answered in [3], by Comfort and Ross, where they have shown that any product of pseudocompact groups is pseudocompact. Thus, finding two countably compact groups whose product is not countably compact would result in two countably compact groups whose product is pseudocompact, but not countably compact. In this spirit, in $\S 5$, we give an example, due to Frolík, of countably compact spaces $X$ and $Y$ whose product is pseudocompact but not countably compact.

The situation for powers of a topological space is, in a sense, reversed. One can characterize spaces all of whose powers are countably compact, as we have done in $\S 2$, but the corresponding question for pseudocompactness, to our knowledge, has not yet been settled. Some partial results in this direction are contained in the next section.

4. Powers and products of pseudocompact spaces. Recall that a space $X$ is pseudocompact if every continuous real-valued function on $X$ is bounded. There is an obvious modification of $\mathscr{D}$-compactness which is suited to the study of pseudocompactness in completely regular spaces. This is because, as Glicksberg observed in [11], a completely regular space $X$ is pseudocompact if, and only if, every sequence of nonempty open subsets of $X$ has a cluster point. (a cluster point of a sequence of sets is a point such that each of its neighborhoods meets infinitely many sets in the sequence). In fact, as Glicksberg shows, it is necessary and sufficient that every sequence of pairwise disjoint, nonempty open sets have a cluster point.

In this section, all spaces are assumed to be completely regular. 
DeFinition 4.1. Let $\mathscr{D}$ be a free ultrafilter on $N$. Let $\left(S_{n}: n \in N\right)$ be a sequence of subsets of a topological space $X$. A point $p \in X$ is called a $\mathscr{D}$-limit point of the sequence $\left(S_{n}: n \in N\right)$ if, for every neighborhood $W$ of $p,\left\{n: S_{n} \cap W \neq \varnothing\right\} \in \mathscr{D}$. A (completely regular) space $X$ is called $\mathscr{D}$-pseudocompact if every sequence of non-empty open subsets of $X$ has a $\mathscr{D}$-limit point.

Making use of arguments similar to those in 2.4, we can readily establish the following facts.

THEOREM 4.2. Every $\mathscr{D}$-pseudocompact space is pseudocompact.

THEOREM 4.3. Every product of $\mathscr{D}$-pseudocompact spaces is $\mathscr{D}$ pseudocompact.

A corollary of these two theorems is that every power of a $\mathscr{D}$ pseudocompact space is pseudocompact. Now, it follows from Theorem 4 of [11], that any product of pseudocompact, locally compact spaces is pseudocompact, and that any product of pseudocompact, first countable spaces is pseudocompact. Since there is no reason, in general, to expect such products to be $\mathscr{D}$-pseudocompact, one cannot hope for a result analogous to Theorem 2.6 for pseudocompact powers. This can be seen in another way. In [11], Glicksberg shows that a product space $\Pi_{\alpha \in I} X_{\alpha}$ is pseudocompact if, and only if, every countable subproduct is pseudocompact. Now, for a sequence of sets $\sigma=$ $\left(S_{n}: n \in N\right)$ in $X$, let $L(\sigma)=\{\mathscr{D} \in \beta N-N: \sigma$ has a $\mathscr{D}$-limit point $\}$. Let $\Sigma$ be the set of sequences (of points) in $X$, and let $\Sigma_{S}$ be the set of all sequences of open subsets of $X$. The proof of 2.6 really shows that every power of $X$ is countably compact if, and only if, for every subset $T$ of $\Sigma, \bigcap_{\sigma \in T} L(\sigma) \neq \varnothing$. Since every power of $X$ is pseudocompact if, and only if, $X^{\aleph^{0}}$ is pseudocompact, we can, in a similar way, conclude that every power of $X$ is pseudocompact if, and only if, for every countable subset $T$ of $\Sigma_{\mathscr{S}}, \bigcap_{\sigma \in T} L(\sigma) \neq \varnothing$.

EXAMPLE 4.4. A space, all of whose powers are pseudocompact which is not $\mathscr{D}$-pseudocompact for any $\mathscr{D}$ in $\beta N-N$.

For each $p$ in $\beta N-N$, let $X_{p}=\beta N-\{p\}$. Let $X=\prod_{p \in \beta N-N} X_{p}$. Since every power of $X$ is a product of locally compact, pseudocompact spaces, every power of $X$ is pseudocompact. But the factor $X_{\mathscr{O}}$ of $X$ is not $\mathscr{D}$-pseudocompact, and so $X$ is not $\mathscr{D}$-pseudocompact for any $\mathscr{D}$ in $\beta N-N$.

4.5. If a space $X$ has a dense subset $D$ such that every sequence 
in $D$ has an accumulation point in $X$, then obviously $X$ is pseudocompact. Many of the familiar examples of pseudocompact spaces have this property, and this criterion for pseudocompactness has been used profitably in many instances. We refer the reader to [2] and [8] for excellent examples of this.

With this in mind, another natural application of $\mathscr{D}$-compactness to the study of pseudocompactness arises. Let us consider spaces $X$ which have a dense subset $A$ such that every sequence in $A$ has a

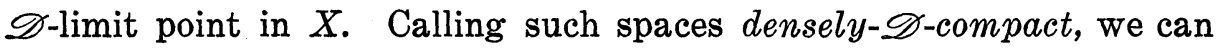
establish the following theorem.

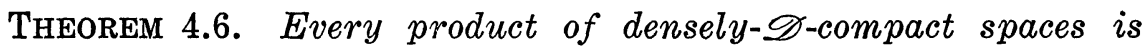

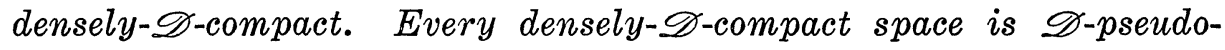
compact.

Proof. The first assertion follows in a straightforward manner, using an argument similar to that in 2.4. To prove the second statement, let $X$ be densely $\mathscr{D}$-compact.

Let $A$ be a dense subset of $X$ such that every sequence in $A$ has a $\mathscr{D}$-limit point in $X$. Now, let $\left(G_{n}: n \in N\right)$ be any sequence of nonempty open sets in $X$. For each $n$, there exists a point $a_{n} \in G_{n} \cap A$. Let $p \in X$ be a $\mathscr{D}$-limit point of the sequence $\left(a_{n}: n \in N\right)$. Then, clearly $p$ is a $\mathscr{D}$-limit point of the sequence $\left(G_{n}: n \in N\right)$. Therefore $X$ is $\mathscr{D}$-pseudocompact.

5. Examples of pseudocompact spaces. In this section we prove several theorems which provide new examples of nontrivial pseudocompact spaces.

Let us first recall the notion of type in $\beta N-N$. The equivalence relation $\sim$ defined on $\beta N-N$ by $x \sim y$ if there exists a homeomorphism of $\beta N$ onto itself taking $x$ to $y$, decomposes $\beta N-N$ into equivalence classes called types. For $p \in \beta N-N, T(p)$ denotes the type of $p$. Recall that, for any $p \in \beta N-N, T(p)$ is dense in $\beta N-N$. (See 6S in [10], and [7].) Note also that every type is a homogeneous space.

We are indebted to Z. Frolík for communicating the following lemma and our first example, and their proofs.

Lemma 5.1 (Frolík). Let $T$ be a type of $\beta N-N$. Then no countable discrete subset of $T$ has a cluster point in $T$. 
Proof. Suppose the statement is false. We shall derive a contradiction. Thus, let $\left(x_{n}: n \in N\right)$ be a discrete subset of a type $T$ which has a cluster point in $T$, say $x$. Find pairwise disjoint, infinite subsets $\left\{A_{n}: n \in N\right\}$ of $N$ such that $N=\bigcup_{n \in N} A_{n}$ and $x_{n} \in \operatorname{cl}_{\beta N} A_{n}$ for each $n$.

Now, for each $n, x_{n}$ and $x$ are of the same type, so we can find, for each $n$, a homeomorphism $f_{n}: \beta N \rightarrow \beta N$ such that $f_{n}\left(x_{n}\right)=x$. Let $g_{n}$ denote the restriction of $f_{n}$ to $A_{n}$. Define $F: N \rightarrow N$ by $F=$ $\mathrm{U}_{n \in N} g_{n}$. Let $F^{\beta}$ denote the Stone-extension of $F$ to $\beta N$. Continuity implies that $F^{\beta}\left(x_{n}\right)=x$ for each $n$, and therefore implies that $F^{\beta}\left(\mathrm{cl}_{\beta N}\left\{x_{n}: n \in N\right\}\right)=\{x\}$. Thus $F^{\beta}(x)=x$.

We now appeal to a result of Katètov, in [3], which implies that the fixed points of $F^{\beta}$ are precisely the points in the $\beta N$-closure of the set of fixed points of $F$. (for a detailed proof, see Lemma 9.1 in [4].) Thus, letting $U=\left\{p \in \beta N: F^{\beta}(p)=p\right\}$, we have $U=\operatorname{cl}_{\beta N}(U \cap N)$. In particular, $U$ is open in $\beta N$. Since $x \in U$, and since $x$ is a cluster point of $\left\{x_{n}: n \in N\right\}$, there is an integer $k$ such that $x_{k} \in U$. For such an integer $k$, we then have to conclude that $x_{k}=F^{\beta}\left(x_{k}\right)=x$.

But this is ridiculous, since $x$ is a cluster point of $\left\{x_{n}: n \in N\right\}$ and $\left\{x_{n}: n \in N\right\}$ is discrete.

We can now present the example, due to Frolík, that was promised in 3.4 .

EXAMPLE 5.2 (Frolík). There exist countably compact spaces $X$ and $Y$ such that $X \times Y$ is pseudocompact and not countably compact.

Proof. Let $T$ be any type of $\beta N-N$. Then every finite power of $N \cup T$ is pseudocompact. (see [2] and [8].) We can find subspaces $P_{1}$ and $P_{2}$ of $\beta N-N$ such that both $X=N \cup T \cup P_{1}$ and $Y=N \cup T \cup P_{2}$ are countably compact, and such that $P_{1} \cap P_{2}=\varnothing$. The proof of the existence of $P_{1}$ and $P_{2}$ is the same as the proof of Lemma 2.9 in [9], and depends on the fact that countably infinite subsets of $\beta N$ have $2^{c}$ points in their closure.

Now the diagonal in $X \times Y$ is $N \cup T$, which is not countably compact by Lemma 5.1. Therefore $X \times Y$ is not countably compact. But since $X \times Y$ contains the dense pseudocompact subspace $(N \cup T) \times$ $(N \cup T), X \times Y$ is pseudocompact.

As was remarked in 4.5 , the pseudocompactness of many familiar spaces can be deduced by the presence of a relatively countably 
compact dense subspace. One of the first examples of a pseudocompact space which has no dense countably compact subspace appears in [14]. The following Theorem 5.3, together with Lemma 5.1, shows there are pseudocompact spaces in which no countable discrete set has a cluster point. Assuming the continuum hypothesis, in 5.4 below, we exhibit a pseudocompact space in which no countable set has a cluster point. It follows that, in all of these examples, there is no dense relatively countably compact subspace. In Theorem 5.6 we show that these spaces have all of their powers pseudocompact. These results show that pseudocompact spaces can be as far from countably compact as is imaginable.

We will show that, if $q$ is a non- $P$-point of $\beta N-N$, then $T(q)$ is pseudocompact. (for the definition and basic properties of $P$-points, see $4 K$ and $4 L$ of [10]). We use the fact that, if $q$ is a non-P-point of $\beta N-N$, there exists a partition $\left\{B_{n}: n \in N\right\}$ of $N$ into infinite sets, such that for each $A \in q$, we have $\left\{n: A \cap B_{n}\right.$ is infinite $\}$ is infinite. This can be shown directly, as in Lemma 9. 14 of [4].

Our original theorem on the pseudocompactness of types held for a more restricted class of types. We are grateful to W. W. Comfort for pointing out that our construction works for all non- $P$-point types.

THeOREM 5.3. If $q$ is a non-P-point of $\beta N-N$, then $T(q)$ is pseudocompact.

Proof. By the result of Glicksberg's quoted earlier, it is sufficient to prove that every sequence of pairwise disjoint, nonempty open subsets of $T(q)$ has a cluster point in $T(q)$.

Thus, let $\left(G_{n}: n \in N\right)$ be a sequence of pairwise disjoint, nonempty open subsets of $T(q)$. For each $n$, there is an infinite subset $A_{n}$ of $N$ such that $\left(\operatorname{cl}_{\beta_{N}} A_{n}\right) \cap T(q) \subseteq G_{n}$. We claim that, for $n \neq m$, $A_{n} \cap A_{m}$ is finite. For, if $A_{m} \cap A_{n}$ was infinite, then $\operatorname{cl}_{\beta_{N}} A_{m} \cap \operatorname{cl}_{\beta_{N}} A_{n}$ would be an open subset of $\beta N$ that meets $\beta N-N$. The density of $T(q)$ would imply that $\left(\mathrm{cl}_{\beta N} A_{m}\right) \cap\left(\mathrm{cl}_{\beta N} A_{n}\right) \cap T(q) \neq \varnothing$, which contradicts the disjointness of $G_{n}$ and $G_{m}$. Thus $n \neq m$ implies that $A_{n} \cap A_{m}$ is finite. For each $n$, let $A_{n}^{\prime}=A_{n}-\mathrm{U}_{i<n} A_{i}$. Then $\left\{A_{n}^{\prime}: n \in \mathrm{N}\right\}$ is a family of pairwise disjoint infinite subsets of $N$ such that $\left(\operatorname{cl}_{\beta_{N}} A_{n}^{\prime}\right) \cap T(q) \leqq G_{n}$ for each $n$. Let $C_{1}=A_{1}^{\prime} \cup\left(N-\bigcup_{n \in N} A_{n}^{\prime}\right)$ and let $C_{n}=A_{n}^{\prime}$ for $n>1$. To find a cluster point of $\left(G_{n}: n \in N\right)$ it clearly suffices to find a cluster point of the sequence $\left(\left(\mathrm{cl}_{\beta_{N}} C_{n}\right) \cap T(q): n \in N\right)$.

We have thus reduced the task of showing $T(q)$ is pseudocompact 
to the following: We must show that, for every partition of $N$ into infinite sets $\left\{A_{n}: n \in N\right\}$, the sequence $\left(\left(\mathrm{cl}_{\beta N} A_{n}\right) \cap T(q): n \in N\right)$ has a cluster point in $T(q)$. To this end, let $N=\bigcup_{n \in N} A_{n}$ be such a partition. Since $q$ is not a $P$-point of $\beta N-N$, there is a partition $N=$ $\mathrm{U}_{n \in N} B_{n}$ of $N$ into infinite sets, such that, for each $A \in q,\left\{n: A \cap B_{n}\right.$ is infinite $\}$ is infinite. Let $f: N \rightarrow N$ be a bijection taking $B_{n}$ onto $A_{n}$ for every $n$. Let $f^{\beta}$ denote its Stone-extension to $\beta N$, and let $p=$ $f^{\beta}(q)$. Then $p \in T(q)$. We claim that $p$ is a cluster point of the sequence $\left(\left(\operatorname{cl}_{\beta N} A_{n}\right) \cap T(q): n \in N\right)$. To prove this, let $\left(\operatorname{cl}_{\beta N} A\right) \cap T(q)$ be any basic neighborhood of $p$ in $T(q)$. Then $A \in p$, and so $f^{-1}(A) \in q$. The set $\left\{n: f^{-1}(A) \cap B_{n}\right.$ is infinite $\}$ is infinite. Since $f$ is a bijection, for infinitely many $n, A \cap A_{n}$ is an infinite set. But for any such $n, \operatorname{cl}_{\beta N} A \cap \operatorname{cl}_{\beta N} A_{n}$ is an open subset of $\beta N$ that meets $\beta N-N$. Since $T(q)$ is dense in $\beta N-N$, for any such $n,\left[\left(\operatorname{cl}_{\beta_{N}} A\right) \cap T(q)\right] \cap\left[\left(\operatorname{cl}_{\beta_{N}} A_{n}\right) \cap\right.$ $T(q)]=\left(\operatorname{cl}_{\beta N} A\right) \cap\left(\operatorname{cl}_{\beta_{N}} A_{n}\right) \cap T(q) \neq \varnothing$. Thus every neighborhood of $p$ in $T(q)$ meets infinitely many of the sets $\left(\operatorname{cl}_{\beta_{N}} A_{n}\right) \cap T(q)$. That is, $p$ is a cluster point of $\left(\left(\mathrm{cl}_{\beta_{N}} A_{n}\right) \cap T(q): n \in N\right)$. As we observed at the beginning of the proof, this enables us to conclude that $T(q)$ is pseudocompact.

REMARK 5.4. In [16], assuming the continuum hypothesis, M. E. Rudin shows there exists a non- $P$-point $q$ in $\beta N-N$ such that $q$ is not in the closure of any countable subset of $\beta N-N$. By our Theorem 5.3, for such $q, T(q)$ is a pseudocompact space in which no countable subset has a limit point. The assumption that $q$ is not a $P$-point in 5.3 is essential, since a pseudocompact $P$-space is finite, and therefore no $P$-point type is pseudocompact.

THEOREM 5.5. Let $q$ be a non-P-point of $\beta N-N$. Then there exists $\mathscr{D}$ in $\beta N-N$ such that $T(q)$ is $\mathscr{D}$-pseudocompact.

Proof. Let $\left\{A_{n}: n \in N\right\}$ be an infinite collection of pairwise disjoint, infinite subsets of $N$ with $N-\mathrm{U}_{n \in N} A_{n}$ infinite. Since $\mathrm{T}(q)$ is pseudocompact, the sequence $\left(\left(\operatorname{cl}_{\beta N} A_{n}\right) \cap T(q): n \in N\right)$ has a cluster point $p \in T(q)$. A proof completely analagous to Lemma 2.2 shows there exists a free ultrafilter $\mathscr{D}$ in $\beta N-N$ such that $p$ is a $\mathscr{D}$-limit point of $\left(\left(\mathrm{cl}_{\beta N} A_{n}\right) \cap T(q): n \in N\right)$. We will show that for this $\mathscr{D}, T(q)$ is $\mathscr{D}$-pseudocompact.

Thus, let $\left(G_{n}: n \in N\right)$ be any sequence of nonempty open subsets of $T(q)$. For each $n$, find an infinite subset $B_{n}$ of $N$ such that $\left(\mathrm{cl}_{\beta_{N}} B_{n}\right) \cap T(q) \subseteq G_{n}$. Using the Disjoint Refinement Lemma 7.5 of [4], we can find a pairwise disjoint sequence $\left(C_{n}: n \in N\right)$ of infinite subsets of $N$, such that $N-\mathrm{U}_{n \in N} C_{n}$ is infinite and $C_{n} \subseteq B_{n}$ for each 
n. It clearly suffices to show that $\left(\left(\mathrm{cl}_{\beta_{N}} C_{n}\right) \cap T(q): n \in N\right)$ has a $\mathscr{D}$ limit point in $T(q)$, for such a point will be a $\mathscr{D}$-limit point of $\left(G_{n}: n \in N\right)$. Now, let $f: N \rightarrow N$ be a bijection taking $A_{n}$ onto $C_{n}$ for every $n$. Let $r=f^{\beta}(p)$. Then $r \in T(q)$. It follows easily that $r$ is a $\mathscr{D}$-limit point of $\left(\left(\operatorname{cl}_{\beta N} C_{n}\right) \cap T(q): n \in N\right)$ in $T(q)$. Thus every sequence of open sets in $T(q)$ has a $\mathscr{D}$-limit point. That is, $T(q)$ is $\mathscr{D}$-pseudocompact.

THEOREM 5.6. If $q$ is a non-P-point of $\beta N-N$, then every power of $T(q)$ is pseudocompact.

Proof. This follows immediately from 5.5, 4.2, and 4.3.

REMARK 5.7. Theorem 5.5 and Lemma 5.1 show that, for a non$P$-point $q$ of $\beta N-N, T(q)$ is an example of a $\mathscr{D}$-pseudocompact space which is not densely $\mathscr{E}$-compact for any $\mathscr{E}$ in $\beta N-N$. Thus the converse of the second statement in 4.6 is false.

5.8. Recall that a completely regular space $X$ is said to be extremally disconnected if every open subset of $X$ has open closure, and basically disconnected if every co-zero set in $X$ has open closure. For the elementary properties of these spaces, the reader is referred to $1 \mathrm{H}$ and $6 \mathrm{M}$ in [10].

THEOREM 5.9. Let $X$ be basically disconnected and locally compact, and let $D$ be a dense subset of $\beta X-X$. Then $X \cup D$ is pseudocompact.

Proof. We will assume not and reach a contradiction. If $X \cup D$ is not pseudocompact, there is an unbounded function $f$ in $C(X \cup D)$. Let $g=\left(f^{2}+1\right)^{-1}$. Then $g \in C(X \cup D), 0<g \leqq 1$ and $\inf _{t \in X \cup D} g(t)=0$. Let $g^{\beta}$ denote the Stone-extension of $g$ to $\beta(X \cup D)=\beta X$. Since $X$ is dense in $X \cup D$, for every $n$ there is a point $x_{n}$ in $X$ such that $g\left(x_{n}\right)<1 / n$. For each $n$, let $G_{n}=\{x \in X: g(x)<1 / n\}$. Since $X$ is locally compact, we can find, for each $n$, a co-zero set $H_{n}$ containing $x_{n}$, whose closure is compact and is contained in $G_{n}$. Let $W=$ $\mathrm{U}_{n \in N} H_{n}$. Then $W$ is a co-zero set in $X$. Now $\mathrm{cl}_{X} W$ is not compact, since the function $g$ on $\mathrm{cl}_{X} W$ does not attain its infimum on $\operatorname{cl}_{X} W$. Thus $\mathrm{cl}_{\beta X} W-X \neq \varnothing$. But, since $X$ is basically disconnected, $\operatorname{cl}_{\beta X} W=$ $\operatorname{cl}_{\beta X}\left(\mathrm{cl}_{X} W\right)$ is open in $\beta X$. Since it meets $\beta X-X$, it must meet $D$, since $D$ is dense in $\beta X-X$. Let $p \in\left(\operatorname{cl}_{\beta X} W\right) \cap D$. Since $g$ is strictly positive on $X \cup D$, there is an integer $m$ such that $g(p)>1 / m$. Let $V=\left\{x \in \beta X: g^{\beta}(x)>1 / m\right\}$. Then $V-\bigcup_{i=1}^{m} \operatorname{cl}_{X} H_{i}$ is a neighborhood of $p$ in $\beta X$ which is disjoint from $W$. This is ridiculous, since $p \in$ 
$\operatorname{cl}_{\beta X} W$. This contradiction proves that $X \cup D$ is pseudocompact.

REMARK 5.10. The conclusion of Theorem 5.9 holds whenever $X$ is locally compact and realcompact. This result was established by Fine and Gillman in Theorem 3.1 of [5]. A special case of 5.9, for extremally disconnected, locally compact spaces, combined with Theorem 3.1 in [21], yields the Fine-Gillman result as a corollary.

\section{REFERENCES}

1. A. R. Bernstein, A new kind of compactness for topological spaces, Fund. Math., 66 (1970), 185-193.

2. W. W. Comfort, A nonpseudocompact product space whose finite subproducts are pseudocompact, Math. Annal., 170 (1967), 41-44.

3. W. W. Comfort and K. A. Ross, Pseudocompactness and uniform continuity in topological groups, Pacific J. Math., 16 (1966), 483-496.

4. W. W. Comfort and S. Negrepontis, The Theory of Ultrafilters, Springer-Verlag, Heidelberg, Grundlehren der math. Wissenschaften, vol. 211, 1974.

5. N. J. Fine and L. Gillman, Remote points in $\beta R$, Proc. Amer. Math. Soc., 13 (1962), 29-36.

6. S. P. Franklin and M. Rajagopalan, Some examples in topology, Trans. Amer. Math. Soc., 155 (1971), 305-314.

7. Z. Frolik, Sums of Ultrafilters, Bull. Amer. Math. Soc., 73 (1967), 87-91.

8. - On two problems of W.W. Comfort, Comm. Math. Univ. Carol. 8, 1 (1967) $139-144$.

9. - The topological product of countably compact spaces, Czech. Math. J., 85 (1960), 329-338.

10. L. Gillman and M. Jerison, Rings of Continuous Functions, Van Nostrand, New York, 1960.

11. I. Glicksberg, Stone-Čech compactifications of products, Trans. Amer. Math. Soc., 90 (1959), 369-382.

12. H. Herrlich and J. van der Slot, Properties which are closely related to compactness, Indag Math., 29 (1967), 524-529.

13. M. Katětov, A theorem on mappings, Comm. Math. Univ. Carol., 8, 3 (1967), 341-433.

14. M. M. Marjanović, A pseudocompact space having no dense countably compact subspace, Glasnik. Mat. Tom. 6 (26)-No. 1-1971, 149-150.

15. N. Noble, Countably compact and pseudocompact products, Czech. Math. J. 19 (1969), 390-397.

16. M. E. Rudin, Partial Orders on the types in $\beta N$, Trans. Amer. Math. Soc., 155, No. 2 (1971), 353-362.

17. V. Saks, Countably Compact Groups, Thesis, Wesleyan University, Middletown, Conn., 1972.

18. V. Saks and R. M. Stephenson Jr., Products of m-compact spaces, Proc. Amer. Math. Soc., 28 (1971), 278-288.

19. C. T. Scarborough and A.H. Stone, Products of nearly compact spaces, Trans. Amer. Math. Soc., 124 (1966), 131-147.

20. A. Weil, Sur les espaces à structure uniforme et sur la topologie générale, Publ. Math. Univ. Strasbourg, Hermann, Paris, 1937.

21. R. G. Woods, Co-absolutes of remainders of Stone-Čech compactifications, Pacific J. Math., 37 (1971), 545-560. 
22. R. G. Woods, Topological extension properties, to appear in Trans. Amer. Math. Soc.

Received April 2, 1974 and in revised form January 20, 1975. The research of the first author was partially supported by a fellowship from the National Research Council of Canada.

UNIVERSITY OF MANITOBA

AND

UNIVERSITY OF COSTA RICA 


\section{PACIFIC JOURNAL OF MATHEMATICS}

\section{EDITORS}

RICHARD ARENS (Managing Editor)

University of California

Los Angeles, California 90024

\section{J. DugundJI}

Department of Mathematics University of Southern California Los Angeles, California 90007

D. Gilbarg and J. Milgram

Stanford University

Stanford, California 94305
University of Washington Seattle, Washington 98105

\section{ASSOCIATE EDITORS}
E. F. BECKENBACH
B. H. NeumanN
F. WolF
K. YoShIDA

\section{SUPPORTING INSTITUTIONS}

\author{
UNIVERSITY OF SOUTHERN CALIFORNIA \\ STANFORD UNIVERSITY \\ UNIVERSITY OF TOKYO \\ UNIVERSITY OF UTAH \\ WASHINGTON STATE UNIVERSITY \\ UNIVERSITY OF WASHINGTON \\ $\stackrel{*}{*} \stackrel{*}{*} \stackrel{*}{ }{ }^{*}$ AMERICAN MATHEMATICAL SOCIETY
}

The Supporting Institutions listed above contribute to the cost of publication of this Journal, but they are not owners or publishers and have no responsibility for its content or policies.

Mathematical papers intended for publication in the Pacific Journal of Mathematics should be in typed form or offset-reproduced, (not dittoed), double spaced with large margins. Underline Greek letters in red, German in green, and script in blue. The first paragraph or two must be capable of being used separately as a synopsis of the entire paper. Items of the bibliography should not be cited there unless absolutely necessary, in which case they must be identified by author and Journal, rather than by item number. Manuscripts, in triplicate, may be sent to any one of the editors. Please classify according to the scheme of Math. Reviews, Index to Vol. 39. All other communications should be addressed to the managing editor, or Elaine Barth, University of California, Los Angeles, California, 90024.

The Pacific Journal of Mathematics expects the author's institution to pay page charges, and reserves the right to delay publication for nonpayment of charges in case of financial emergency.

100 reprints are provided free for each article, only if page charges have been substantially paid. Additional copies may be obtained at cost in multiples of 50 .

The Pacific Journal of Mathematics is issued monthly as of January 1966. Regular subscription rate: $\$ 72.00$ a year (6 Vols., 12 issues). Special rate: $\$ 36.00$ a year to individual members of supporting institutions.

Subscriptions, orders for back numbers, and changes of address should be sent to Pacific Journal of Mathematics, 103 Highland Boulevard, Berkeley, California, 94708.

\section{PUBLISHED BY PACIFIC JOURNAL OF MATHEMATICS, A NON-PROFIT CORPORATION}

Printed at Kokusai Bunken Insatsusha (International Academic Printing Co., Ltd.), 270, 3-chome Totsuka-cho, Shinjuku-ku, Tokyo 160, Japan.

\section{Copyright (C) 1975 by Pacific Journal of Mathematics} Manufactured and first issued in Japan 


\section{Pacific Journal of Mathematics}

\section{Vol. 57, No. $2 \quad$ February, 1975}

Norman Larrabee Alling, On Cauchy's theorem for real algebraic curves with boundary .......

Daniel D. Anderson, A remark on the lattice of ideals of a Prüfer domain ..................

Dennis Neal Barr and Peter D. Miletta, A necessary and sufficient condition for uniqueness of

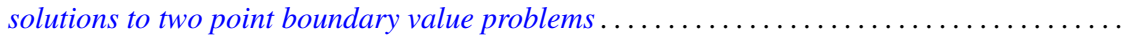

Ladislav Beran, On solvability of generalized orthomodular lattices . . . . . . . . . . ........

L. Carlitz, A three-term relation for some sums related to Dedekind sums . . . . . . . . . .....

Arthur Herbert Copeland, Jr. and Albert Oscar Shar, Images and pre-images of localization

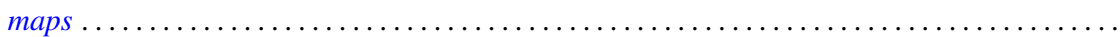

G. G. Dandapat, John L. Hunsucker and Carl Pomerance, Some new results on odd perfect

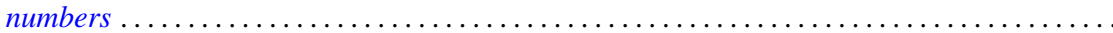

M. Edelstein and L. Keener, Characterizations of infinite-dimensional and nonreflexive

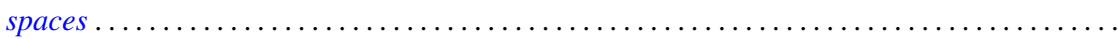

Francis James Flanigan, On Levi factors of derivation algebras and the radical embedding

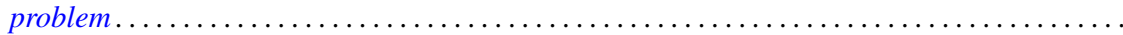

Harvey Friedman, Provable equality in primitive recursive arithmetic with and without

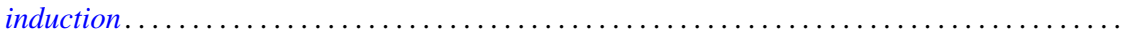

Joseph Braucher Fugate and Lee K. Mohler, The fixed point property for tree-like continua with

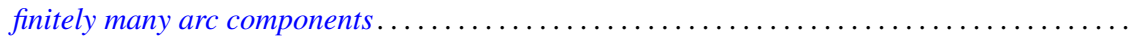

John Norman Ginsburg and Victor Harold Saks, Some applications of ultrafilters in

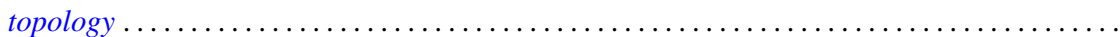

Arjun K. Gupta, Generalisation of a "square" functional equation .....................

Thomas Lee Hayden and Frank Jones Massey, Nonlinear holomorphic semigroups ..........

V. Kannan and Thekkedath Thrivikraman, Lattices of Hausdorff compactifications of a locally

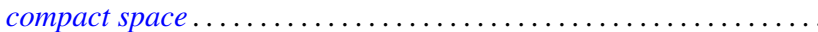

J. E. Kerlin and Wilfred Dennis Pepe, Norm decreasing homomorphisms between group

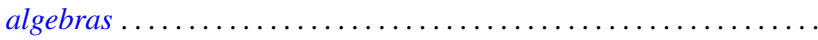

Young K. Kwon, Behavior of $\Phi$-bounded harmonic functions at the Wiener boundary ...

Richard Arthur Levaro, Projective quasi-coherent sheaves of modules .

Chung Lin, Rearranging Fourier transforms on groups...........................

David Lowell Lovelady, An asymptotic analysis of an odd order linear differential equation . . 4475

Jerry Malzan, On groups with a single involution .......................... 481

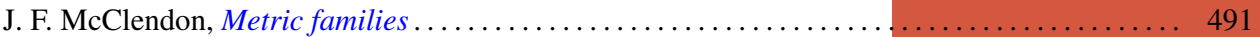

Carl Pomerance, On multiply perfect numbers with a special property .

Mohan S. Putcha and Adil Mohamed Yaqub, Polynomial constraints for finiteness of semisimple rings. .

Calvin R. Putnam, Hyponormal contractions and strong power convergence . . . . . . . . . 531

Douglas Conner Ravenel, Multiplicative operations in $\mathrm{BP} * \mathrm{BP} \ldots \ldots \ldots \ldots \ldots \ldots \ldots \ldots \ldots .539$

Judith Roitman, Attaining the spread at cardinals which are not strong limits . . . . . . . . . 545

Kazuyuki Saitô, Groups of *-automorphisms and invariant maps of von Neumann algebras . . . 553

Brian Kirkwood Schmidt, Homotopy invariance of contravariant functors acting on smooth

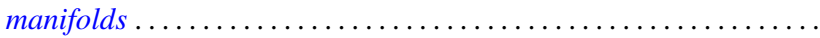

Kenneth Barry Stolarsky, The sum of the distances to $N$ points on a sphere.

Mark Lawrence Teply, Semiprime rings with the singular splitting property.

J. Pelham Thomas, Maximal connected Hausdorff spaces..............

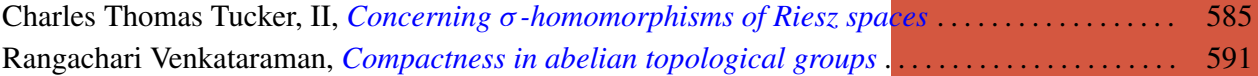

William Charles Waterhouse, Basically bounded functors and flat sheaves . . . . . . . . . . . 597

David Westreich, Bifurcation of operator equations with unbounded linearized part ......... 611

William Robin Zame, Extendibility, boundedness and sequential convergence in spaces of 